\title{
Influence of the substorm precipitation and polar cap patches on GPS signals at high latitudes
}

\author{
V. B. Belakhovsky ${ }^{1}$, Y. Jin ${ }^{2}$, W. Miloch ${ }^{2}$ \\ ${ }^{1}$ Polar Geophysical Institute, Apatity, 184209, Russia \\ ${ }^{2}$ Department of Physics, University of Oslo, Oslo, Norway \\ E-mail: belakhov@mail.ru
}

\begin{abstract}
The comparative research of the influence of substrorm precipitation and polar cap patches (PCP) on the GPS signals disturbances in the polar ionosphere was done. For this aim we use the GPS scintillation receivers at Ny-Ålesund, operated by the University of Oslo. The presence of the auroral particle precipitation and polar cap patches was determined by using data from the EISCAT $42 \mathrm{~m}$ radar on Svalbard. We consider tens of events when the simultaneous EISCAT $42 \mathrm{~m}$ and GPS data were available. We demonstrate that substorm-associated precipitations can lead to a strong GPS phase $\left(\sigma_{\varphi}\right)$ scintillations up to $\sim 2$ radians which is much stronger than those usually produced by PCPs. At the same PCPs can lead to strong ROT (rate of total electron content) variations. So, our observations suggest that the substorms and PCPs, being different types of the high-latitude disturbances, lead to the development of different types and scales of ionospheric irregularities.
\end{abstract}

Accepted: 15.09 .2020

DOI: 10.21046/2070-7401-2020-17-6-139-144

\section{Introduction}

The Global Navigation Satellite Systems (GNSS) become more important for modern society. Among the different GNSS GPS is a mostly used for the ionosphere studies because there are a lot of 2-frequency GPS receivers all over the world. The ionosphere as a medium for the radio waves propagation can have a negative influence on the quality of received signal. Irregularities in the plasma density distribution can lead to fast fluctuations of amplitude and phase of the signal which is referred to as ionosphere scintillations [1]. The strong scintillations reduce the quality of the signal and even lead to the signal loss. Thus, the investigation of GPS scintillations is an important aspect of space weather. The level of scintillations is characterized by the phase $\left(\sigma_{\Phi}\right)$ and amplitude $\left(S_{4}\right)$ scintillation indexes.

Amplitude scintillations are caused by the plasma irregularities with scale sizes ranging from tens to hundreds of meters, while the phase scintillations are caused by the irregularities with the sizes from hundreds of meters to several kilometers. Ionospheric scintillations are most severe in the equatorial region and at high latitudes [2].

The most severe disturbances in polar ionosphere are substorms and polar cap patches. Polar cap patches are 100-1000 km islands of enhanced plasma density being segmented from the dayside highdensity plasma in the cusp region [3]. There are a lot of mechanisms of the polar cap patches formation [4]. During strong and stable polar cap convection, segmentation may not happen and a continuous tongue of ionization (TOI) may be formed across the polar cap.

A magnetospheric substorm is a transient process initiated on the nightside of the Earth, in which a significant amount of energy derived from the solar wind-magnetosphere interaction is deposited in the auroral ionosphere and magnetosphere [5]. 
In paper [6] it was found that polar cap patches have their biggest impact on GPS signals once they reach the nightside auroral oval, in particular when combined with upward field-aligned currents. It is shown in the paper [7] that PCP can produce GPS scintillations quite comparable with scintillations during the particle precipitation with appearance of strong green aurora.

In the present work we address the following question: substorm particle precipitation or polar cap patches have stronger impact on the scintillations of GPS signals.

\section{Data used}

The Ny-Ålesund (NYA) GPS scintillation receiver of the University of Oslo (UiO) was the main instrument used in our study. Upon availability of data, the Skibotn (Norway, mainland) GPS receiver was also used. The UiO GNSS scintillation receiver is the standard GNSS Ionospheric Scintillation/ total electron content (TEC) Monitor (GISTM), model GSV4004B [8]. The carrier phase and power at the $\mathrm{L} 1$ frequency $(1.57542 \mathrm{GHz})$ are tracked and recorded at $50 \mathrm{~Hz}$ rate. The phase $\left(\sigma_{\varphi}\right)$ and amplitude scintillation indices $\left(S_{4}\right)$ are also calculated and recorded automatically. The phase scintillation index is defined as the standard deviation of the carrier phase that has been detrended by the high-pass sixth-order Butterworth filter with a cutoff frequency of $0.1 \mathrm{~Hz}$. The amplitude scintillation index $\left(S_{4}\right)$ is defined as the standard deviation of the received signal power, based on the $50-\mathrm{Hz}$ sampling rate, normalized to the average signal power over one minute periods. The phase scintillation index is defined as the standard deviation of the carrier phase that has been detrended by a high-pass sixth-order Butterworth filter that were computed over one minute intervals are used in this study. The GPS TEC data have been postprocessed by using WinTEC-P [9]. The ROT (Rate of TEC) data over 1 min are also used to depict the TEC variations, where ROT $=\Delta \mathrm{TEC} / \Delta \mathrm{t}$.

For the describing the ionospheric plasma parameters (density, ion and electron temperature, line of sight ion velocity as a function of range) we used the Svalbard EISCAT $42 \mathrm{~m}$ radar. The beam of the EISCAT $42 \mathrm{~m}$ radar is directed along the geomagnetic field (azimuth $=184^{\circ}$, elevation $=82^{\circ}$ ). IMAGE magnetometer data was used for the geomagnetic field observations. OMNI database was used for the evaluating the solar wind and interplanetary magnetic field parameters.

\section{Data analysis}

In the present study, we considered the influence of nighttime substorm precipitation and polar cap patches on the GPS scintillations. We focused mainly on the phase scintillation index because amplitude scintillation index $\left(S_{4}\right)$ practically has no large variations at high latitudes. The presence of the particle precipitation into the ionosphere associated with the appearance of the aurora was determined as the density increase between 100-200 km altitudes according to the EISCAT radar data. The presence of the polar cap patches was determined as a strong density increase above $200 \mathrm{~km}$ altitude. We consider mainly the winter scintillations because the EISCAT radar works most often in winter season. In general, we identified about a hundred of different cases for years 2010-2017 when the data from the EISCAT $42 \mathrm{~m}$ radar was available, however, in this paper we present only typical examples. The presented conclusions are valid for the common picture. The some analyse was done before in the paper [10], this paper is the continuation of the paper [10].

\subsection{Substorm precipitation}

The example of the substorm precipitation and the GPS scintillations response to it is shown in figure 1 (11 December 2015). It was observed two substorms during this day. The first one was at 15:3017:00 UT, the second one was at 20:00-22:00 UT. It was polar substorms because it mainly observed at latitude higher than $70^{\circ}$.

It can be noticed that the amplitude of the first substorm reaches the value about $1400 \mathrm{nT}$ at Hornsund (HOR) station, at NAL station the amplitude of the substorm was $600 \mathrm{nT}$. The second substorm was has lower amplitude than first substorm (600 nT at HOR station). These substorms was observed without geomagnetic storm $(\mathrm{SYM}-\mathrm{H} \approx-10 \mathrm{nT})$, however the solar wind speed was quite high $(V=640-680 \mathrm{~km} / \mathrm{s})$ according to the OMNI database.

The ionosphere plasma density increases during substorm on more that order from $9 \cdot 10^{4}$ to $1 \cdot 10^{6} \mathrm{~cm}^{-3}$ at altitudes $100-160 \mathrm{~km}$. The substorms were accompanied by the strong increase of aurora intensity in different spectrum lines (not shown). 


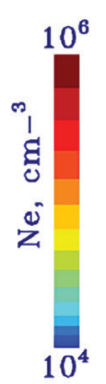

11 December 2015. Svalbard, 42m.
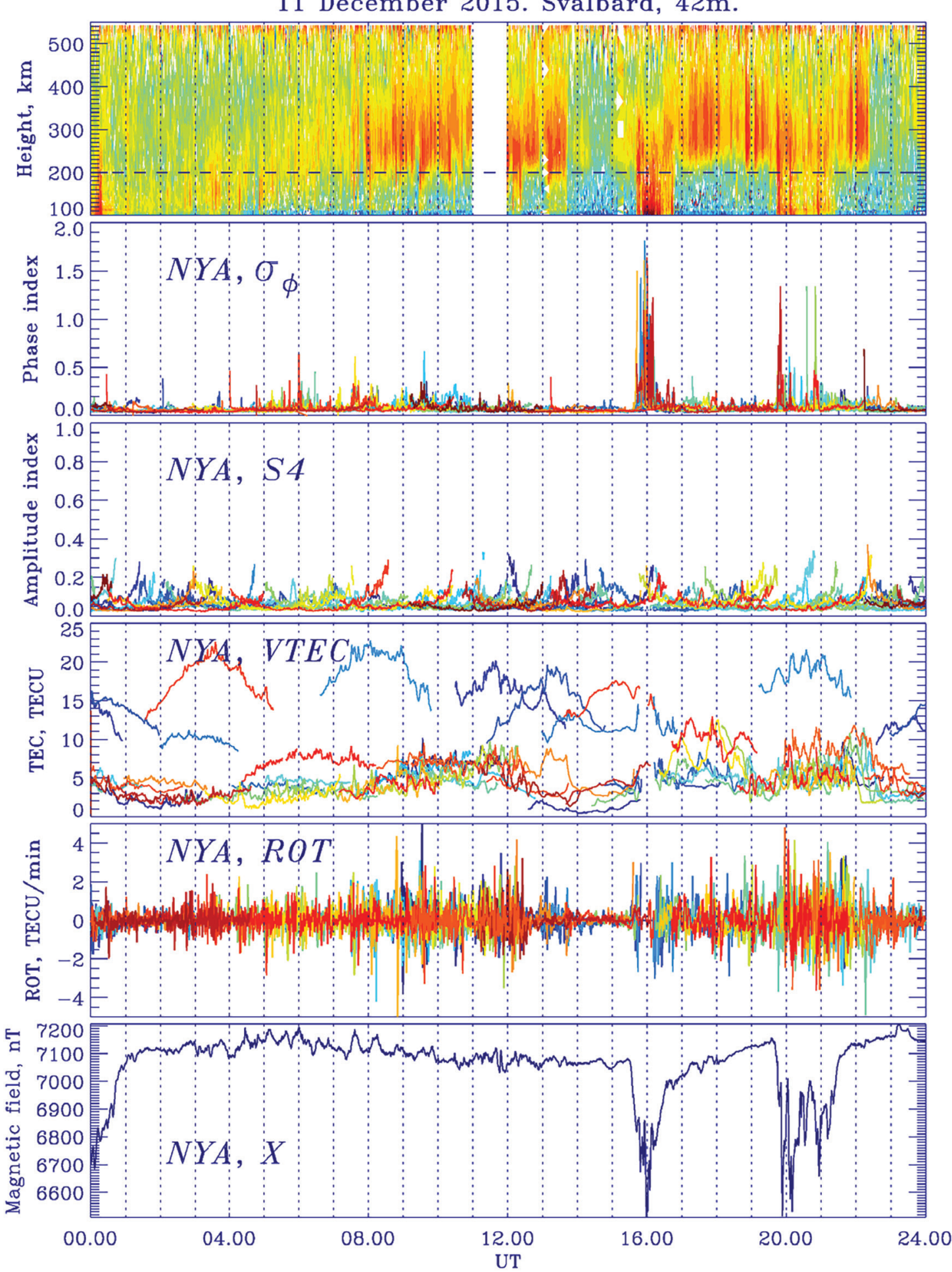

Figure 1. Ionosphere plasma density according to the EISCAT $42 \mathrm{~m}$ radar data; phase scintillation index and amplitude scintillation index according to the GPS receiver at NYA station; TEC and ROT variations according to the GPS receiver at NYA station; geomagnetic field variations (X-component) at NYA station for the 11 December 2015.

The phase index reaches the value about 2 radians approximately during the first substorm. The growth of the phase scintillation index was seen not mainly during the substorm expansion phase (30-40 minutes). During the second substorm the phase scintillation index has the lower value (0.5-1.5 radians). The growth of the phase scintillation index was seen as sharp increases during 5-10 minutes time intervals. 
Substorms do not lead to the great TEC increase. It is seen the absence of the TEC data during the substorm. It testifies about the phase failure of GPS signal. The ULF waves in Pi3 frequency range embedded into the substorm structure can have contribution into the particle acceleration into the ionosphere which leads to such strong values of the phase scintillation indexes.

\subsection{Polar cap patches}

The example of the evening-nighttime polar cap patches (PCP) is shown on figure 2 for the 10 February 2015.

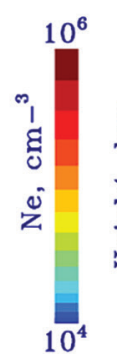

10 February 2015. Svalbard, 42m.

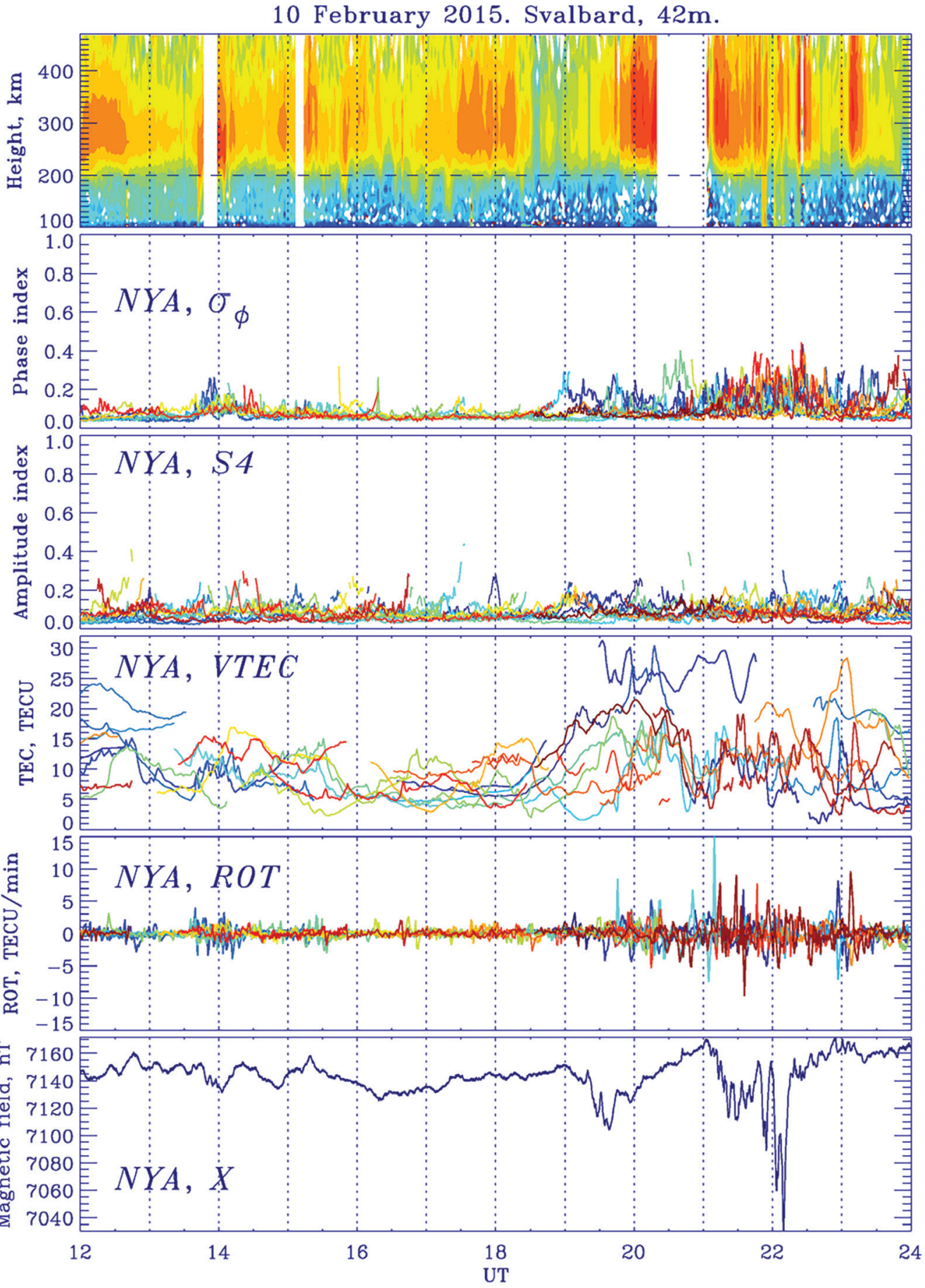

Figure 2. Ionosphere plasma density according to the EISCAT $42 \mathrm{~m}$ radar data; phase scintillation index and amplitude scintillation index according to the GPS receiver at NYA station; TEC and ROT variations according to the GPS receiver at NYA station; geomagnetic field variations (X-component) at NYA station for the 10 February 2015. 
The PCP was observed at 19:00-23:30 UT as a density increase above $200 \mathrm{~km}$ according to the EISCAT data. At NYA GPS receiver the phase scintillation index reaches the medium value ( 0.4 radians). However, the ROT variations for the PCP reach the high values (10-15 TECU/min).

During the PCP appearance the Bz-component of IMF has negative values $(-6 \mathrm{nT})$ during 3 hours. It leads to the development of the small substorm. The amplitude of the substorm is 120-140 nT in X-component of the geomagnetic field at NYA station. The PCP is also identified in the aurora intensity variations as forms propagating from the polar to low latitudes in $630.0 \mathrm{~nm}$ (red line) emission (not shown) at 19:00-23:00 UT.

It was done the comparison of the PCP development on EISCAT $42 \mathrm{~m}$ radar (Svalbard) and UHF radar located at lower latitudes in Tromso (not shown). The sharp increase of the plasma density from 12 to 17 UT above $200 \mathrm{~km}$ is caused by the sunlight. At latitudes of SKN (TRO) stations the PCP manifests itself as a long lasting substorm (more than 4 hours duration) with the amplitude $200-250 \mathrm{nT}$. At SKN stations the phase scintillation index has approximately the same values $(0.4-$ 0.5 radians) as in polar latitudes.

For the all consider PCP cases phase index has the value less than 1.

\section{Conclusions}

The influence of substorms and polar cap patches on perturbations of GPS signals with using receivers on Svalbard and in Skiboth is considered. Substorms (even without PCP) lead to the maximum values of the phase scintillation index (1.5-2 radians). The growth of the phase index observed mainly during the substorm expansion phase. ULF waves in Pi3 frequency range during a substorm producing auroral arcs can lead to such high values of the phase index.

Polar cap patches leads to the prolonged variations of phase index with smaller values (less than 1 ). At the same time polar cap patches can lead to strong ROT variations $(10-15 \mathrm{TECU} / \mathrm{min})$ in comparison with the substorms disturbances. So, our observations suggest that the substorms and PCPs, being different types of the high-latitude disturbances, lead to the development of different types and scales of ionospheric irregularities.

\section{Acknowledgements}

This study is supported by the grant of the Russian Science Foundation (Project No. 18-77-10018).

The authors thank the Norwegian Polar Research Institute at Ny-Ålesund for assisting us with the GPS receiver in Ny-Ålesund, Bjørn Lybekk and Espen Trondsen for the instrument operations. The IMF data are provided by the NASA OMNI Web service (http://omniwegsfc.nasa.gov).

The authors wish to thank IMAGE (http://www.ava.fmi.fi/image/), EISCAT groups for the available data. EISCAT is an international association supported by research organizations in China (CRIRP), Finland (SA), Japan (NIPR and STEL), Norway (NFR), Sweden (VR), and the United Kingdom (NERC). Data from EISCAT can be obtained from the Madrigal database http://www.eiscat.se/madrigal.

The University of Oslo ASI data are available at http://tid.uio.no/plasma/aurora. The IMF and solar wind data were provided by the NASA OMNI Web service (http://omniweb.gsfc.nasa.gov).

\section{References}

[1] Basu S., Groves K. M., Basu Su., Sultan P. J., Specification and forecasting of scintillations in communication/navigation links: Current status and future plans, J. Atmospheric and Solar-Terrestrial Physics, 2002, Vol. 64(16), pp. 1745-1754.

[2] Kintner P. M., Ledvina B. M., de Paula E.R., GPS and ionospheric scintillations, Space Weather, 2007, Vol. 5(9), 09003, 23 p.

[3] Oksavik K., Barth V. L., Moen J, Lester M., On the entry and transit of high-density plasma across the polar cap, J. Geophysical Research, 2010, Vol. 115(A2), A12308, 15 p.

[4] Moen J., Oksavik K., Alfonsi L., Daabakk Y., Romano V., Spogli L., Space weather challenges of the polar cap ionosphere, J. Space Weather Space Climate, 2013, Vol. 3, A02, 13 p., DOI: 10.1051/swsc/2013025.

[5] Rostoker G., Akasofu S.-I., Foster J., Greenwald R., Kamide Y., Kawasaki K., Lui A., McPherron R., Russelland C., Magnetospheric substorms - Definition and signatures, J. Geophysical Research, 1980, Vol. 85(A4), pp. 1663-1668, DOI: 10.1029/JA085iA04p01663. 
[6] Clausen L. B.N., Moen J. I., Hosokawa K., Holmes J. M., GPS scintillations in the high latitudes during periods of dayside and nightside reconnection, J. Geophysical Research Space Physics, 2016, Vol. 121, pp. 3293-3309, DOI: 10.1002/2015JA022199.

[7] Jin Y., Moen J. Miloch W., GPS scintillation effects associated with polar cap patches and substorm auroral activity: direct comparison, J. Space Weather and Space Climate, 2014, Vol. 4, A23, 6 p., DOI: 10.1051/ swsc/2014019.

[8] Van Dierendonck A.J., Klobuchar J., Hua Q., Ionospheric Scintillation Monitoring Using Commercial Single Frequency C/A Code Receivers, 6 Intern. Technical Meeting of the Satellite Division of The Institute of Navigation, Proc., 1993, pp. 1333-1342.

[9] Carrano C.S., Anghel A., Quinn R.A., Groves K. M., Kalman filter estimation of plasmaspheric total electron content using GPS, Radio Science, 2009, Vol. 44(1), RS0A10, 14 p., DOI: 10.1029/2008RS004070.

[10] Belakhovsky V. B., Jin Y., Miloch W.J., Influence of Different Ionospheric Disturbances on the GPS Scintillations at High Latitudes, Springer Proc. in Earth and Environmental Sciences, 2019, pp. 281-287, DOI: $10.1007 / 978-3-030-21788-4 \_24$. 